\title{
Analysis of a reactive agility field test
}

\author{
Warren B. Young*, Ben Willey \\ School of Human Movement and Sport Sciences, University of Ballarat, Australia \\ Received 12 March 2009; received in revised form 13 May 2009; accepted 13 May 2009
}

\section{Introduction}

In team sports agility is an important quality to evade opponents when attacking or to place pressure on opponents when defending. Agility has traditionally been thought of as simply the ability to change direction quickly. However over 30 years ago, it was recognised that agility can be a complex quality containing temporal and spatial uncertainly. ${ }^{1}$ More recently it has been suggested that agility contains both a change of direction movement and a perceptual and decision making component, since changes of direction and speed are often performed in response to an opponent's actions. ${ }^{2,3}$

Reactive agility has been tested in netball by requiring players to react and change direction in response to a video clip of an attacking player passing a ball. ${ }^{4}$ Highly skilled players produced significantly faster movement times and decision times than less skilled players, the latter defined as

\footnotetext{
* Corresponding author.

E-mail address: w.young@ballarat.edu.au (W.B. Young).
}

the time between the instant of ball release from the video display to the instant of foot plant to change direction.

An alternative field test of reactive agility was designed for Australian Rules football ${ }^{5}$ and later used for rugby league. 6,7 This test required the tested athlete to respond to a live tester who performed pre-determined side-step movements to the left or right. The test was found to have adequate reliability and to distinguish between higher and lower performance groups. ${ }^{5,6}$ The studies on rugby league ${ }^{6,7}$ also recorded the test with high speed video $(200 \mathrm{~Hz})$ to identify the decision time, defined as the time from final foot plant of the tester to foot plant of the player pushing off to change direction. A limitation of these studies is that they did not isolate movement time from decision time. Rather they defined "movement time" as the total time of the test, which included the tester's movements, the decision time and the movement time of the athlete.

Therefore the purpose of the present study was to determine the influence of tester time, decision time and the athlete's response movement time to provide further insights into some factors that contribute to agility performance as well as the efficacy of this field test of reactive agility. 


\section{Methods}

The participants were 31 male Australian Rules football players from a semi-professional Victorian Football League (VFL) club. The mean \pm SD age, height and body mass was $21.5 \pm 1.9$ years, $184.8 \pm 8.0 \mathrm{~cm}$ and $83.0 \pm 8.7 \mathrm{~kg}$, respectively. The participants were free from injury at the time of testing, provided informed consent and the study was approved by the University's Human Research Ethics Committee.

Within 1 week before assessment, the participants were provided with information about the test and performed eight practice trials. The reactive agility test layout and procedures were the same as previously used with rugby league players $^{6,7}$ incorporating eight trials presented in a random order. A single tester who was experienced in agility movements provided the change of direction stimulus to all players. Four foot patterns were performed by the tester, and have been described previously. ${ }^{5,6}$

The total time for the agility test was recorded with a dual beam infra-red timing light system (Speedlight Timing System, Swift Performance Equipment). The time commenced when the tester moved forward and departed from a light gate and finished when the participant ran through a stop gate to the left or right after he had changed direction. This total time was comprised of the tester's movements, the time required by the participant to decide which direction the tester was going to move, and finally the time taken by the participant to change direction and sprint to the finish gate. These components of the total time were determined by counting frames from video recordings. A high speed digital video camera (Redlake PCI 2000S) operating at $125 \mathrm{~Hz}$ was positioned behind and to the side of the tester so that the field of view could clearly identify the placement of the feet of both the tester and participant for all trials. The following times were obtained:

1. Total time (described above from the timing light system).

2. Tester time was the time from the first forward movement of the tester when the body left the beam to the instant the foot was planted for the final side-step.

3. Decision time was the time from the instant the tester planted his foot for the side-step to change direction to the instant the participant planted his foot to change direction. Although the participant must produce some movement after the decision has been made prior to his foot plant, the instant of foot plant was required to develop an operational definition of decision time. This meant that a negative value was possible when the participant planted his foot to change direction before the tester planted his foot to produce the stimulus.

4. Response movement time was the time from the instant the participant planted his foot to change direction to the instant of running through the finish gate.

Based on the definitions of these components, the total time was equal to the sum of the tester time, decision time
Table 1

Descriptive results for the total time and its components.

\begin{tabular}{lrr}
\hline & Mean \pm SD & Percent of total time \\
\hline Total time $(\mathrm{ms})$ & $1967 \pm 84$ & \\
Tester time $(\mathrm{ms})$ & $552 \pm 25$ & 28.0 \\
Decision time (ms) & $70 \pm 57$ & 3.6 \\
Response movement time (ms) & $1345 \pm 52$ & 68.4 \\
\hline
\end{tabular}

and response movement time. The means of the eight trials for all measures were retained for analysis.

Pearson correlations were computed to determine the relationships among the test variables. The coefficient of variation $(\mathrm{CV})$ was calculated to determine the variability associated with the tester times.

\section{Results}

Table 1 shows the means and standard deviations for the total agility time and the three components with the corresponding percentage contribution to the total time. The correlation matrix describing the relationships between the times is indicated in Table 2. Of the three components that make up the total time, decision time had the highest correlation $(r=0.77, p=0.00)$ with the total time. The $\mathrm{CV}$ for the tester times was $5.1 \%$.

\section{Discussion}

Approximately two thirds of the total time was comprised of a side-step movement to change direction and sprint to the stop gate (response movement time) and tester movement averaged $28 \%$ of the total time. An important finding was that although decision time was less than $4 \%$ of the total time, the correlation between decision time and total time was quite high $(r=0.77, p=0.00)$. Further, this correlation with total time was greater than for the response movement time $(r=0.59)$ or tester time $(r=0.37)$, indicating that decision time was the most influential of the test components for explaining the variability in total time. This is consistent with the findings of previous research indicating that the speed of decision making is important for reactive agility. ${ }^{4,7}$ It is likely that the individuals who performed well in the reactive agility test were able to use advanced cues from the tester's side-step movements to quickly anticipate the directional change. This is supported by the finding that five of the players achieved negative decision times, which indicates

Table 2

Pearson correlation coefficients with the $\mathrm{p}$ values are shown in brackets.

\begin{tabular}{lllr}
\hline & Tester time & Decision time & \multicolumn{1}{l}{$\begin{array}{l}\text { Response } \\
\text { movement time }\end{array}$} \\
\hline Total time & $0.37(p=0.04)$ & $0.77(p=0.00)$ & $\begin{array}{r}0.59(p=0.00) \\
\text { Tester time }\end{array}$ \\
Decision time & & $0.26(p=0.16)$ & $\begin{array}{r}-0.17(p=0.38) \\
0.03(p=0.88)\end{array}$ \\
\hline
\end{tabular}


that they had planted their foot to change direction before the tester had planted his foot to side-step. Four of these players were ranked within the group in the top eight in total time, suggesting their anticipatory skill contributed to their good agility performance.

One way to look at the influence of the tester time is to describe the variability of this time presented to the athletes. The CV of $5.1 \%$ is relatively low but the variability may be enough to make a meaningful difference to the total time. For example, the longest mean tester time for one subject was $596 \mathrm{~ms}$ and the shortest was $455 \mathrm{~ms}$. This difference of $141 \mathrm{~ms}$ represents $7 \%$ of the total time. Since the tester used in this study was an experienced team sport player and had practised the four footwork patterns, it is unlikely that the tester time variability could be significantly reduced by the use of a different tester. The small positive correlation between tester time and total time $(r=0.37, p<0.05)$ indicates that although the variation in tester movement time is not large, it can potentially make a meaningful difference to the mean total time recorded by an athlete in a series of trials.

\section{Conclusions}

Decision time was found to have the strongest relationship with the total time, suggesting perceptual skills can potentially influence agility performance. Future research in Australian Rules football should attempt to determine what specific cues from an attacking player's movements and body position provide the most useful anticipatory information about the ensuing change of direction. It should also determine if perceptual skills can be trained to enhance decision-making speed and accuracy. Tester time can have a meaningful influence over the total test score for an individual.

\section{Practical implications}

- Since tester time can influence the total time recorded in the reactive agility test, it should be isolated from high speed video recordings. The tester time could be potentially removed by subtracting it from the total time.

- Decision time has a strong influence on total time (agility performance) and therefore perceptual skill should be addressed in agility testing and training.

- The very low correlation between decision time and response movement time indicated that some players had relatively good decision times but did not produce fast movements, and vice-versa. Training should be individualised in an attempt to emphasise the development of the player's weakness.

\section{Acknowledgments}

The authors would like to thank Gerard Fitgerald and Stephen Abbruzzese for their assistance. There was no financial support for this project.

\section{References}

1. Chelladurai P. Manifestations of agility. Can Assoc Health PE Rec 1976;42:36-41.

2. Young WB, James R, Montgomery I. Is muscle power related to running speed with changes of direction? J Sports Med Phys Fit 2002;43: 282-8.

3. Sheppard JM, Young WB. Agility literature review: classification, training and testing. J Sports Sci 2006;24:919-32.

4. Farrow D, Young W, Bruce L. The development of a test of reactive agility for netball: a new methodology. J Sci Med Sport 2005;8: 40-8.

5. Sheppard JM, Young WB, Doyle TA, Sheppard TA, Newton RU. An evaluation of a new test of reactive agility, and its relationship to sprint speed and change of direction speed. J Sci Med Sport 2006;9: 342-9.

6. Gabbett T, Kelly J, Sheppard J. Speed, change of direction speed, and reactive agility of rugby league players. J Strength Cond Res 2008;22(1):174-81.

7. Gabbett T, Benton D. Reactive agility of rugby league players. J Sci Med Sport 2009;12:212-4. 